\title{
Pattern-Dependent Role of NMDA Receptors in Action Potential Generation: Consequences on Extracellular Signal-Regulated Kinase Activation
}

\author{
Meilan Zhao, J. Paige Adams, and Serena M. Dudek \\ National Institute of Environmental Health Sciences, National Institutes of Health, Research Triangle Park, North Carolina 27709
}

\begin{abstract}
Synaptic long-term potentiation is maintained through gene transcription, but how the nucleus is recruited remains controversial. Activation of extracellular signal-regulated kinases (ERKs) 1 and 2 with synaptic stimulation has been shown to require NMDA receptors (NMDARs), yet stimulation intensities sufficient to recruit action potentials (APs) also appear to be required. This has led us to ask the question of whether NMDARs are necessary for AP generation as they relate to ERK activation. To test this, we examined the effects of NMDAR blockade on APs induced with synaptic stimulation using whole-cell current-clamp recordings from CA1 pyramidal cells in hippocampal slices. NMDAR antagonists were found to potently inhibit APs generated with 5 and $100 \mathrm{~Hz}$ synaptic stimulation. Blockade of APs and ERK activation could be overcome with the addition of the $\mathrm{GABA}_{\mathrm{A}}$ antagonist bicuculline, indicating that APs are sufficient to activate signals such as ERK in the nucleus and throughout the neuron in the continued presence of NMDAR antagonists. Interestingly, no effects of the NMDAR antagonists were observed when theta-burst stimulation (TBS) was used. This resistance to the antagonists is conferred by temporal summation during the bursts. These results clarify findings from a previous study showing that ERK activation induced with TBS is resistant to 2-amino-5-phosphonovalerate, in contrast to that induced with 5 or $100 \mathrm{~Hz}$ stimulation, which is sensitive. By showing that NMDAR blockade inhibits AP generation, we demonstrate that a major role that NMDARs play in cell-wide and nuclear ERK activation is through their contribution to action potential generation.
\end{abstract}

Key words: long-term potentiation; transcription; translation; cAMP response element-binding protein; Elk-1; synapse-to-nucleus signal

\section{Introduction}

An area of debate in the study of long-term potentiation (LTP) concerns how signals originating in the dendrites lead to altered RNA transcription in the nucleus. One model proposes that biochemical signals, such as the extracellular signal-regulated kinases (ERKs) 1 and 2, travel from the potentiated synapse(s) to the nucleus, where they can then induce gene transcription (for review, see Deisseroth et al., 2003). This idea is supported by evidence showing that in long-term facilitation induced in Aplysia neurons, ERK translocates to the nucleus (Martin et al., 1997). An alternative model postulates that action potential firing in the postsynaptic neuron can induce nuclear changes via increases in intracellular calcium and increases in the cell-wide activation of ERK (Dudek and Fields, 2002). Notably, ERK phosphorylates several transcription factors, thus potentially playing a role in gene transcription (Caboche et al., 2001) and late-phase LTP (Rosenblum et al., 2002).

Activation of ERK has been shown to be exquisitely sensitive

\footnotetext{
Received Feb. 14, 2005; revised April 21, 2005; accepted May 13, 2005.

This work was supported by the National Institute of Environmental Health Sciences. We thank Devin Smith for his work on the histology and members of the Laboratory of Neurobiology for critical reading of this manuscript.

Correspondence should be addressed to Serena M. Dudek, National Institute of Environmental Health Sciences, National Institutes of Health, P.0. Box 12233, Maryland F2-04, Research Triangle Park, NC 27709. E-mail: dudek@niehs.nih.gov.

DOI:10.1523/JNEUROSCI.1579-05.2005

Copyright $\odot 2005$ Society for Neuroscience $\quad$ 0270-6474/05/257032-08\$15.00/0
}

to regulation by neuronal activity. Effective regulators of ERK include glutamate receptor activation (Bading and Greenberg, 1991), depolarization with potassium (Baron et al., 1996), LTPand long-term depression (LTD)-inducing stimulation (English and Sweatt, 1996; Dudek and Fields, 2001; Thiels et al., 2002), and learning (Blum et al., 1999). In the context of synaptic stimulation at 5-100 Hz, ERK activation can be entirely prevented with NMDA receptor (NMDAR) blockade (English and Sweatt, 1996; Dudek and Fields, 2001), leading to the conclusion that NMDARs are critical to ERK activation by synaptic activity. The requirement for stimulation intensities sufficient to recruit action potentials (Dudek and Fields, 2001) could therefore be attributable to a necessity of action potentials to achieve maximal opening of NMDAR channels and recruitment of enzymes upstream of ERK. However, in one stimulation paradigm [theta burst stimulation (TBS)], the increase in immunostaining for activated, phosphorylated ERK (p-ERK) is resistant to NMDAR antagonists; only when both NMDARs and L-type calcium channels are blocked is the staining, and hence ERK activation, significantly reduced (Dudek and Fields, 2001). An explanation for this difference between one LTP-inducing stimulus $(100 \mathrm{~Hz})$ and another (TBS) is that TBS could be more likely than the $100 \mathrm{~Hz}$ (or $5 \mathrm{~Hz}$ ) stimulation to recruit voltage-sensitive calcium channels (VSCCs). An alternative explanation is that NMDARs could play a critical role in action potential generation and that the stimulation pattern is important in determining how much or how little 
a role. Supporting this idea is the observation that NMDAR antagonists can inhibit cell firing in the visual system (Miller et al., 1989; Sillito et al., 1990; Blitz and Regehr, 2003) (but see Bear et al., 1990) and in the hippocampus, as assessed by population spike size (Abraham and Mason, 1988; Burgard et al., 1989; Dahl et al., 1990).

Here, we show that NMDARs do play a critical role in action potential generation in the hippocampal slice and that this role is dependent on stimulation pattern. To further evaluate whether ERK could be activated under NMDAR blockade when action potentials are controlled, we asked whether restoring action potentials with bicuculline (bic) could similarly restore ERK activation in the presence of NMDAR blockers. Our results demonstrate that action potentials, possibly through VSCCs or other sources of calcium, are sufficient to support ERK activation in the presence of NMDAR blockade, consistent with a previous study showing that antidromically induced action potentials, in the absence of synaptic activity, are sufficient for ERK activation and LTP-related signaling (Dudek and Fields, 2002). These results suggest that NMDARs are necessary for synaptically induced ERK signaling in the nucleus, primarily because of their ability to contribute to action potential generation, and that stimulation pattern strongly influences this contribution.

\section{Materials and Methods}

Slice preparation. Rats, 11-21 d of age, were overdosed with injected sodium pentobarbital $(50 \mathrm{mg} / \mathrm{kg}$, i.p.) before decapitation. Brains were then removed, and the hippocampi were sliced at $350 \mu \mathrm{m}$ with a vibrating microtome in ice-cold oxygenated sucrose-substituted artificial CSF (ACSF) (Richardson and Messer, 1995). Freshly cut slices were transferred to a storage chamber filled with oxygenated normal ACSF containing the following (in mM): $124 \mathrm{NaCl}, 2.5 \mathrm{KCl}, 2 \mathrm{CaCl}_{2}, 1.5 \mathrm{MgCl}_{2}, 10$ glucose, $26 \mathrm{NaHCO}_{3}$, and $1.25 \mathrm{NaH}_{2} \mathrm{PO}_{4}$, $\mathrm{pH} 7.35,300-310 \mathrm{mOsm}$, at room temperature for $\geq 1 \mathrm{~h}$ before any recording. Although recordings were performed at room temperature, similar results were obtained at $34^{\circ} \mathrm{C}$. D-(-)-2-amino-5-phosphonovaleric acid (D-APV), (+)-5-methyl10,11-dihydro-5 $\mathrm{H}$-dibenzo [a,d] cyclohepten-5,10-imine maleate (MK801), carboxypiperazin-4-yl-propyl-1-phosphonic acid (CPP), and bicuculline were purchased from Tocris Cookson (Ellisville, MO). For immunocytochemistry and Western blot analysis, slices were prepared as described above, except that the slices from 6- to 12-week-old rats were cut in normal ACSF with $1 \mathrm{~mm} \mathrm{CaCl}, 3 \mathrm{mM} \mathrm{MgCl}_{2}$, and $5 \mathrm{~mm}$ kynurenic acid. For Western blot analysis, area CA1 was microdissected to make "mini-slices" of area CA1 while slices were still in this ACSF. Slices (either whole for immunocytochemistry or mini-slices for Western blot analysis) were then placed immediately in the recording chamber at $34^{\circ} \mathrm{C}$ in normal ACSF containing $2.5 \mathrm{~mm} \mathrm{CaCl}$ and $1.5 \mathrm{~mm} \mathrm{MgCl}$.

Patch-clamp electrophysiology. Electrodes pulled from glass capillaries (Garner Glass, Claremont, CA) were filled with an intracellular solution composed of the following (in $\mathrm{mm}$ ): $10 \mathrm{KCl}, 120 \mathrm{~K}^{+}$gluconate, 40 HEPES, $3 \mathrm{MgCl}_{2}, 0.5$ EGTA, 2 ATP, and 0.3 GTP, pH 7.2, 275-280 mOsm, with a final resistance of $4-6 \mathrm{M} \Omega$. Cells were visualized with a microscope fitted with differential interference contrast optics and a water-immersion $40 \times$ objective. Whole-cell current-clamp recordings from hippocampal CA1 pyramidal cells were performed. Only cells with stable access resistance throughout the recording were used; recordings from cells with unstable membrane currents were discarded, as were neurons with a resting membrane potential less than $-55 \mathrm{mV}$ (average, $-61.0 \mathrm{mV}$ ). Action potentials were evoked with stimulation to the stratum radiatum using a cluster-type bipolar stimulating electrode (Frederick Haer Company, Bowdoinham, ME) in the vicinity of the recorded CA1 pyramidal cells. Stimulation intensity $(\approx 80-150 \mu \mathrm{A})$ was adjusted to induce firing of several action potentials when $5 \mathrm{~Hz}$ was used. Thetaburst stimulation consisted of $10100-\mathrm{Hz}$ bursts of four pulses delivered at $5 \mathrm{~Hz}$ (Larson et al., 1986). Recordings were filtered at $2 \mathrm{kHz}$, digitized at $5-10 \mathrm{kHz}$ using the Digidata $1322 \mathrm{~A}$ digitizer, and acquired and ana- lyzed using pClamp 8.1 software (Molecular Devices, Union City, CA). Data values are expressed as means \pm SEM. In some cases, action potential amplitudes were truncated for figure clarity.

Field recording electrophysiology. Slices were prepared, and physiology was performed according to Dudek and Fields (2001), except that stimulation was delivered with a duration of $130 \mu$ s.

Immunocytochemistry. Immunocytochemistry was performed as described previously (Dudek and Fields, 2001). Briefly, stimulated slices were fixed and resectioned to $30 \mu \mathrm{m}$ on a freezing microtome. Sections were permeabilized, blocked, and incubated in primary antibody against the active, dually phosphorylated ERK1/2 (Promega, Madison, WI) at 1:2000. Sections were then incubated with secondary antibody, followed by processing for visualization using a DAB reaction product (Vector Elite ABC; Vector Laboratories, Burlingame, CA). Positive staining appears dark. Staining induced with $5 \mathrm{~Hz}$ was no more (or less) robust than that induced with $100 \mathrm{~Hz}$, a finding that is confirmed on Western blots (data not shown). Because we cannot match the number of (postsynaptic) action potentials between the different stimulation frequencies, we do not make direct comparisons between p-ERK staining induced in the $5 \mathrm{~Hz}, 100 \mathrm{~Hz}$, or TBS cases. When possible, comparisons are made between slices from the same animal. In cases in which bicuculline was used, the NMDAR antagonist was applied before the bicuculline to reduce the possibility that bursting or spontaneous activity would influence the results.

Western blot analysis. Mini-slices were removed from the chamber and flash-frozen on dry ice $3 \mathrm{~min}$ after stimulation. For each condition, 10 mini-slices were pooled. The nuclei from the mini-slices were isolated using the CellLytic NuCLEAR Extraction Kit (Sigma, St. Louis, MO), with the following modifications. Frozen mini-slices were homogenized on ice in $500 \mu \mathrm{l}$ of $1 \times$ hypotonic buffer with $0.6 \%$ Igepal CA- $630,1 \mu \mathrm{M}$ dithiothreitol, $1 \times$ protease inhibitor mixture (all provided in the kit), with the addition of $5 \mathrm{~mm}$ EGTA, $1 \times$ phosphatase inhibitor mixture set II (Calbiochem, La Jolla, CA), and $1 \mu \mathrm{m}$ okadaic acid (Sigma). The homogenate was then centrifuged at $9000 \times g$ in a Microfuge for $20 \mathrm{~min}$ at $4^{\circ} \mathrm{C}$. The supernatant containing the cytosolic fraction of the cells was then boiled in sample buffer for $10 \mathrm{~min}$. The pellet, containing the nuclei from the cells, was resuspended in sample buffer and boiled for $10 \mathrm{~min}$. Samples were resolved by SDS-PAGE and transferred to polyvinylidene difluoride membrane. The membranes were then incubated with the same active, dually phosphorylated ERK1/2 (Promega) antibody used in the immunocytochemistry experiments. Proteins were then detected using enhanced chemiluminescence.

\section{Results}

NMDA receptor blockade inhibits action potential generation In view of the finding that activation of ERK with electrical stimulation in hippocampal slices requires intensities that evoke action potentials (Dudek and Fields, 2001), we thought it was critical to determine the nature of NMDAR contribution to the following: do NMDARs work specifically at the level of the synapse to activate ERK cell-wide, or is cell-wide ERK activation instead mediated through an NMDAR contribution to action potential generation? The hypothesis that NMDARs play a dominant role in activating ERK by supporting action potential firing can be readily tested by examining the effects of NMDAR antagonists on action potential generation. Whole-cell current-clamp recordings were made from pyramidal CA1 neurons to determine the number of action potentials fired during 5 or $100 \mathrm{~Hz}$ synaptic stimulation of the stratum radiatum. The effects of the competitive NMDAR antagonist APV on action potential generation are shown in Figure 1. Thirty traces were recorded during stimulation at $5 \mathrm{~Hz}$ before, during, and after washout of $50 \mu \mathrm{M}$ APV (only eight are shown superimposed for figure clarity). Treatment of the cells with the drug led to an abrupt elimination of action potentials, which was readily reversed after washout (Fig. 1A) (control, $13.78 \pm 2.61$; APV, $0.22 \pm 0.21$; washout, $11.44 \pm 2.17 ; n=10)$. To test effects of APV on action potential 


\section{A $5 \mathrm{~Hz}$ Stimulation \pm APV $(50 \mu \mathrm{M})$}
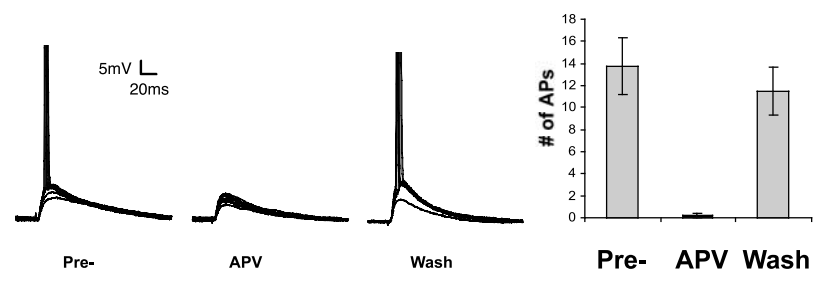

B $100 \mathrm{~Hz}$ Stimulation \pm APV $(50 \mu M)$
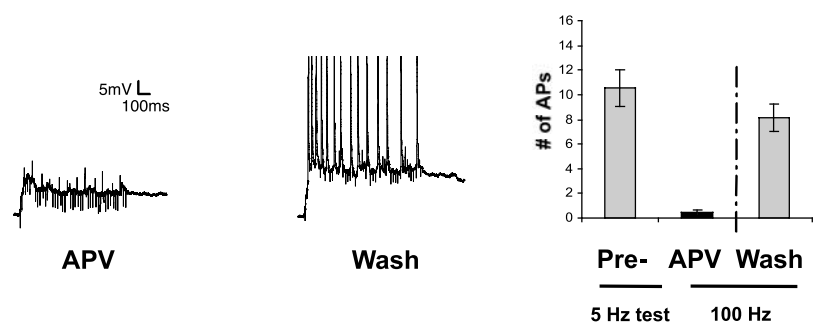

C 5 or $100 \mathrm{~Hz}$ Stimulation \pm MK801 $(20 \mu \mathrm{M})$
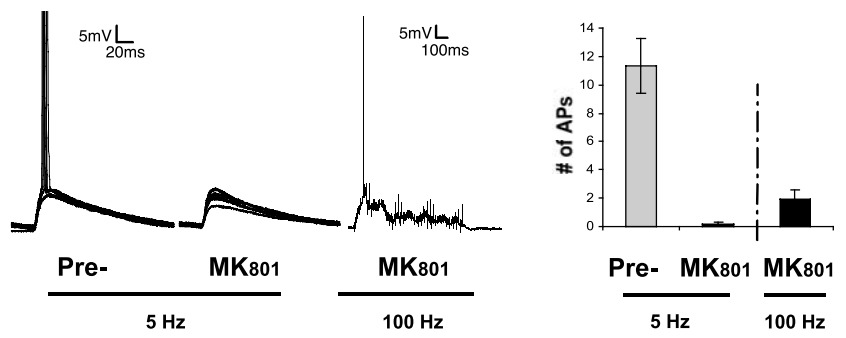

Figure 1. Bath application of APV or MK-801 reduces the number of action potentials generated in response to 5 and $100 \mathrm{~Hz}$ synaptic stimulation. $A$, Recordings from a representative cell in response to stimulation at $5 \mathrm{~Hz}$ before (Pre-), during (APV), and after (Wash) washout of APV $(50 \mu \mathrm{m}) .0 \mathrm{f} 30$ traces recorded in each condition, only eight are shown superimposed for clarity. $B$, Recordings from a representative cell in response to $100 \mathrm{~Hz}$ stimulation in the presence of APV and after washout (Wash). Compiled data from the same experiments are shown to the right of traces from each frequency. In the $100 \mathrm{~Hz}$ case, a $5 \mathrm{~Hz}$ test stimulation was used to assess washin of the drug and the number of action potentials in the presence of drug compared with that in the absence of drug (washout). Means \pm SEMs from 5-10 CA1 pyramidal neurons are shown. C, Left, Recordings from a representative cell in response to stimulation at $5 \mathrm{~Hz}$ before and during application of MK-801 (20 $\mu \mathrm{M})$. Of 30 traces recorded, only eight are shown superimposed for clarity. Middle, Recordings from the same cell in response to $100 \mathrm{~Hz}$ stimulation in the presence of MK-801. Right, Compiled data from the same experiments. Five hertz stimulation was used to assess action potentials in the predrug condition, and the action potentials were induced with either 5 or $100 \mathrm{~Hz}$ in the presence of drug. Means \pm SEMs from 10 CA1 pyramidal neurons are shown. Washout of MK-801 was not assessed. Error bars represent SEMs.

number using $100 \mathrm{~Hz}$ stimulation without inducing LTP in the "predrug" condition, $5 \mathrm{~Hz}$ stimulation was used to assess the washin of the drug, and the number of action potentials induced with $100 \mathrm{~Hz}$ stimulation during the drug application was compared with the number after the drug had been washed out. The results were similar to those obtained with $5 \mathrm{~Hz}$ stimulation: APV severely reduced action potential generation in response to 100 $\mathrm{Hz}$ synaptic stimulation (Fig. $1 B$ ) (APV, $0.44 \pm 0.24$; washout, $8.13 \pm 1.14 ; n=8)$. Similarly, another NMDAR antagonist, CPP $(20 \mu \mathrm{M})$, also led to the reversible elimination of action potentials induced with 5 and $100 \mathrm{~Hz}$ stimulation (supplemental Fig. 1, available at www.jneurosci.org as supplemental material). Action potentials induced with these frequencies were blocked at concentrations that were still selective, in that they did not appear to impact AMPA receptor-dependent currents (supplemental Fig. 2, available at www.jneurosci.org as supplemental material) and are the concentrations typically used by many experimenters. The general effect (APV and CPP blockade of action potentials) was not unique to slices from young animals in that both drugs were effective in slices from older animals $(5 \mathrm{~Hz}, 6$ weeks of age, control, $13.25 \pm 3.32,50 \mu \mathrm{M}$ $\mathrm{APV}, 0.25 \pm 0.25, n=4$; 4 weeks of age, control, $14.0 \pm 2.51$, $20 \mu \mathrm{M} \mathrm{CPP,} 1.33 \pm 1.33, n=3$ ).

The compounds CPP and APV are both competitive antagonists at the glutamate-binding site on the NMDAR. To rule out the possibility that the drugs were acting nonspecifically at other glutamate receptors, we tested whether a similar effect could be observed with MK-801, a use-dependent blocker of the NMDARchannel complex. The results were comparable with those observed with APV and CPP: bath application of MK-801 resulted in the elimination of action potentials (Fig. 1C) (5 Hz control, $11.35 \pm 1.90 ; 5 \mathrm{~Hz}$ MK801, $0.20 \pm 0.13 ; n=10)$. Several $5 \mathrm{~Hz}$ episodes were used before the final recordings to facilitate the use-dependent actions of MK-801. Because MK-801 cannot be readily washed out, $5 \mathrm{~Hz}$ stimulation was used to assess washin of the drug, and the $100 \mathrm{~Hz}$ condition was measured only during drug application (Fig. 1C) $[100 \mathrm{~Hz}$ average control (data not shown), $7.84 \pm 0.97, n=19$ vs $100 \mathrm{~Hz}$ MK801, $1.9 \pm 0.67, n=$ 10]. MK-801 delivered to the inside of the cell via the pipette solution was found to be equally effective, ruling out a role for presynaptic NMDARs in action potential generation (supplemental Fig. 3, available at www.jneurosci.org as supplemental material). Together, these results demonstrate that action potentials induced with synaptic stimulation at 5 or $100 \mathrm{~Hz}$ can be blocked with competitive and noncompetitive NMDAR antagonists acting at postsynaptic NMDARs.

\section{Restoring action potentials restores ERK activation}

If action potentials are in fact the critical factor mediating ERK activation, restoration of action potentials in the presence of NMDAR blockade should restore ERK activation and, hence, immunostaining for p-ERK. Attempts to fully overcome NMDAR antagonist inhibition of action potentials with increased stimulus intensity generally failed; although the strategy could do so partially, it often resulted in the instability of the cells athigh-stimulus intensities (supplemental Fig. 4, available at www. jneurosci.org as supplemental material). A more effective strategy involved the use of the $\mathrm{GABA}_{\mathrm{A}}$ receptor antagonist bicuculline. At $10 \mu \mathrm{M}$, bicuculline was very effective at overcoming the inhibition of action potentials by NMDAR blockade at both 5 and $100 \mathrm{~Hz}$ stimulation (Fig. $2 \mathrm{~A}, B)(5 \mathrm{~Hz}$ control, $10.07 \pm 2.01 ; 5 \mathrm{~Hz}$ CPP plus bic, $14.50 \pm 2.53 ; 100 \mathrm{~Hz}$ CPP plus bic, $7.82 \pm 1.77 ; n=$ 14). Notably, when action potentials were restored with bicuculline (in APV or CPP), staining for phospho-ERK was also restored to control or greater levels (Fig. $3 A, B$ ). As observed previously, NMDAR blockade effectively eliminated both dendritic and somatic staining for p-ERK induced with $5 \mathrm{~Hz}$ (Dudek and Fields, 2001) and $100 \mathrm{~Hz}$ (English and Sweatt, 1996) synaptic stimulation in Western blots. Consistent with this, staining in the dendrites as well as in the cell bodies was restored when bicuculline was used with the NMDAR antagonist. This result was confirmed using immunoblot analysis; as evident in both cytosolic and nuclear fractions, APV was ineffective at blocking ERK acti- 
A

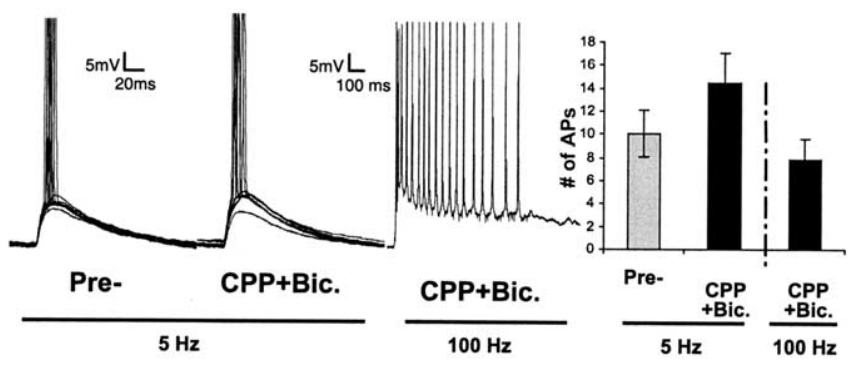

Figure 2. Bicuculline restores action potentials blocked by CPP. Bath application of bicuculline $(10 \mu \mathrm{M})$ prevents the loss of action potentials with (PP $(20 \mu \mathrm{M})$. $\boldsymbol{A}$, Recordings from a representative cell in response to 5 and $100 \mathrm{~Hz}$ stimulation before (Pre-) and during (CPP + Bic.) application of bicuculline and CPP. $\boldsymbol{B}$, Compiled data from the same experiments showing that action potentials are restored in both 5 and $100 \mathrm{~Hz}$ stimulation cases. Means \pm SEMs from 14 CA1 pyramidal neurons are shown. Error bars represent SEMs.

vation when bicuculline was present (Fig. 3C). Bicuculline alone did not increase activation of p-ERK in the control condition, demonstrating that its actions are not caused by elevation of basal ERK activation (Fig. 3C). However, bicuculline did increase the number of cells stained and the intensity of staining in response to stimulation when compared with stimulation in untreated slices (data not shown). This is likely to be a result of the fact that more cells were recruited to fire, and the cells that did fire, fired more spikes. Together, these results are consistent with the hypothesis that NMDAR antagonists interfere with ERK activation by blocking action potentials, because the staining was fully restored in parallel with the restoration of action potential generation in the continued presence of NMDAR antagonists.

\section{Action potentials evoked with TBS are resistant to APV and CPP}

The sensitivity of ERK activation to NMDAR blockers is dramatically different between two different LTP-inducing stimuli; 100 $\mathrm{Hz}$ is sensitive, whereas TBS is not. Therefore, our results suggested that the sensitivity of action potential generation to NMDAR blockers could also be profoundly different between the two. To test this, we used the same approach as described above. As predicted, we observed that TBS-induced action potentials were completely insensitive to blockade of NMDARs at concentrations that blocked action potentials at 5 and $100 \mathrm{~Hz}$ (Fig. $4 A-C$ ) (TBS in APV, $5.08 \pm 1.05$; washout, $5.62 \pm 0.10 ; n=13$; TBS in CPP, $4.22 \pm 1.05$; washout, $4.33 \pm 1.11 ; n=13$ ). A partial block of TBS-induced action potentials was observed with much higher concentrations of CPP and APV (Fig. 5A,B), which may exhibit nonselective activity and, as such, are not typically used in this range. Lower drug concentrations (for example, 50-100 $\mu \mathrm{M}$ APV) still block LTP induced with TBS (Larson and Lynch, 1988) without blocking action potentials.

Why are the action potentials induced with TBS resistant to NMDAR blockade, when action potentials evoked with 5 and 100 $\mathrm{Hz}$ are not? One possibility is that within the $100 \mathrm{~Hz}$ bursts in TBS, temporal summation is sufficient to support action potential firing without NMDAR contribution and, additionally, lacks the rundown of synaptic responses observed with unpatterned $100 \mathrm{~Hz}$ stimulation. To test the idea that temporal summation is important for the resistance of TBS-evoked action potentials to NMDAR antagonists, we varied the interval between individual synaptic pulses (within bursts) and determined whether longer intervals resulted in increased sensitivity to $50 \mu \mathrm{M}$ APV. Consistent with the hypothesis that temporal summation is important for NMDAR- independent action potential generation, we found that increasing the interpulse interval (within the bursts) from 10 $\mathrm{ms}$ to 20 and $40 \mathrm{~ms}$ increased the susceptibility of action potentials to the APV; action potential numbers were severely curtailed at intervals of $40 \mathrm{~ms}$ (Fig. 6 A) $(10 \mathrm{~ms}, 5.38 \pm 0.49 ; 20 \mathrm{~ms}, 3.5 \pm$ $0.39 ; 40 \mathrm{~ms}, 1.58 \pm 0.30 ; n=11)$. In contrast, varying the interburst interval (between bursts) had no effect on the action potential firing (Fig. $6 B$ ) $[200 \mathrm{~ms}(5 \mathrm{~Hz}), 5.57 \pm 0.63 ; 333 \mathrm{~ms}(3 \mathrm{~Hz})$, $5.39 \pm 0.60 ; 500 \mathrm{~ms}(2 \mathrm{~Hz}), 5.52 \pm 0.65 ; n=10]$, indicating that the frequency of burst delivery was not responsible for this unique feature of the TBS. These data demonstrate that withinburst intervals, but not between-burst intervals, confer resistance of action potential generation to NMDAR antagonists when slices are stimulated with TBS.

\section{Discussion}

Previous work has shown that NMDARs can be important in cell firing, both in the visual system (Miller et al., 1989; Sillito et al., 1990; Blitz and Regehr, 2003) and in the dentate gyrus of the hippocampus (Abraham and Mason, 1988; Burgard et al., 1989; Dahl et al., 1990). The consequences of NMDAR inhibition on action potential generation in hippocampal neurons using LTPinducing stimulation, however, have not been studied previously. Here, we show that NMDARs play a particularly important role in determining whether neurons will fire in response to synaptic stimulation, including LTP-inducing stimulation such as $100 \mathrm{~Hz}$. This role in action potential generation has important implications regarding the interpretation of pharmacological studies looking at cell-wide ERK activation; blockade of NMDARs results in a loss of $\mathrm{p}$-ERK staining that can now be directly tied to a blockade of action potentials. Two conditions in which action potentials were not blocked in the presence of antagonists, bicuculline or TBS, show that when action potentials are preserved, ERK activation remains intact.

Because the effect of these antagonists can be rapidly reversed on washout, NMDAR blockade appears to be an acute effect, distinct from the NMDAR-dependent persistence of adenosinemediated bursting in CA3 (Thummler and Dunwiddie, 2000). Given our results, it is also interesting to note that rhythmic oscillations and bursts evoked with synaptic stimulation are inhibited with NMDAR antagonists (Bonansco et al., 2002). Curiously, our dose-response curves for the effect of NMDAR antagonists on action potential generation (Fig. 5) are shifted rightward by nearly a factor of 10 when compared with published affinities of these drugs for the NMDAR (Benveniste et al., 1990). This suggests that a significant number of NMDARs need to be blocked before any partial effects can be seen on action potential number. Maximal effects on action potentials (at 5 and $100 \mathrm{~Hz}$ ) occur at concentrations that appear to be in the selective ranges of the drugs (i.e., no apparent effect on AMPA receptors) (supplemental Fig. 2, available at www.jneurosci.org as supplemental material) and therefore cannot be explained simply as nonselective effects of the drugs on AMPA receptors. Effects on presynaptic NMDARs can also be ruled out, given our observation that intracellular MK-801 also blocked action potential generation. In addition, nonspecific drug effects on sodium channels are also unlikely, given that bicuculline can restore action potential generation in the continued presence of NMDAR antagonists. Repetitive stimulation such as that used here is apparently unnecessary for the role of NMDARs in action potential firing, given 
that single population spikes are also sensitive to NMDAR antagonists in the dentate gyrus (Abraham and Mason, 1988; Burgard et al., 1989; Dahl et al., 1990).

Using mice instead of rats, one study found that action potentials (population spikes) induced with $5 \mathrm{~Hz}$ stimulation were insensitive to APV at $100 \mu \mathrm{M}$ (Thomas et al., 1998). Given our observation that higher-stimulation intensities reduced the effects of NMDAR antagonists (supplemental Fig. 4, available at www.jneurosci. org as supplemental material), it is likely that this discrepancy is attributable to differences in the relative effectiveness of electrical stimulation between mice and rats or perhaps in stimulus intensity used in the two studies. This is supported by the observation that $80 \%$ of the EPSPs recorded with $5 \mathrm{~Hz}$ stimulation evoked spikes (Thomas et al., 1998), whereas $<50 \%$ of the EPSPs evoked action potentials in our study. If the AMPA receptor component of the EPSPs was sufficiently large, NMDARs would not be necessary for action potential firing.

Interestingly, although bicuculline had no effect on basal ERK activation (Fig. 3C), we did observe increased staining with stimulation when bicuculline was used. We found this to be wholly consistent with our assertion that action potentials are important for ERK activation, in that as more action potentials are evoked, a larger number of cells and wider area are stained. An increase in intensity could simply be attributable to the cells firing more action potentials for a given stimulus. If NMDARs were contributing something substantial in addition to calcium, we would expect to see a larger effect of the antagonists, at least in the dendrites, but we do not.

Why are action potentials induced with TBS resistant to blockade of NMDARs? A likely explanation, which is supported by our finding that short within-burst intervals are important for antagonist resistance, is that the temporal summation of the AMPA receptor currents occurring during the $(100 \mathrm{~Hz})$ bursts is sufficient to support action potential firing without NMDAR involvement (Figs. 6, 7). Action potentials induced with TBS occurred in response to approximately one of every two bursts, both with and without NMDAR antagonists, and thus do not appear at rates higher than those induced with $5 \mathrm{~Hz}$ synaptic stimulation. Other mechanisms, such as inhibitory tone, neuromodulatory action, or repetitive stimulation are more likely to be responsible for any bursting of postsynaptic CA1 neurons that may occur during the theta rhythm (present study, but not observed consistently). An interesting contrast can be made with another LTP-inducing stimulation, $100 \mathrm{~Hz}$, in which typically only one to two spikes are fired, if any,

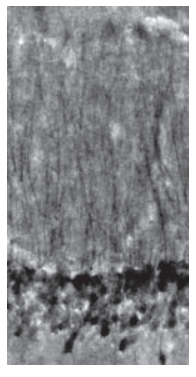

Control

$5 \mathrm{~Hz}$

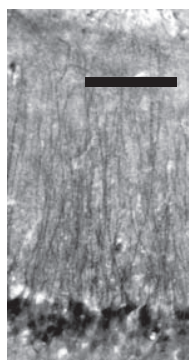

Control $100 \mathrm{~Hz}$
C

\section{A $5 \mathrm{~Hz}$ Stimulation}

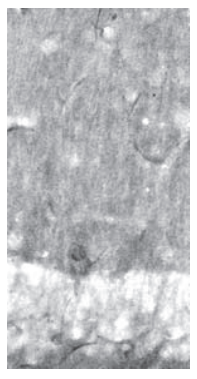

+APV
+ APV
+ Bic.

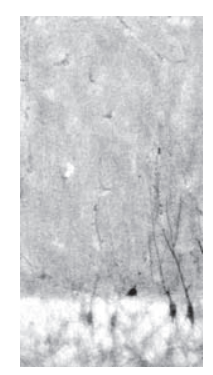

$+\mathrm{CPP}$

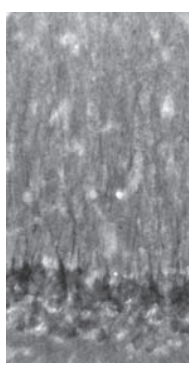

+CPP

+ Bic.

\section{B $100 \mathrm{~Hz}$ Stimulation}

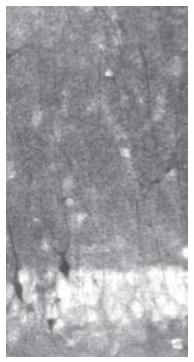

+APV

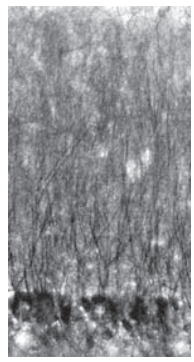

+APV

+ Bic.

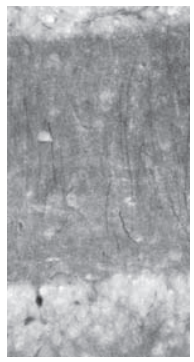

+CPP

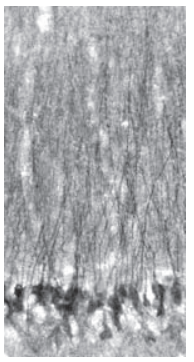

+ CPP

+ Bic.

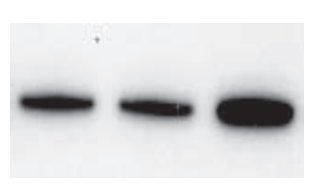

C $\mathrm{C}+\mathrm{Bic} 5 \mathrm{~Hz}+\mathrm{Bic}$

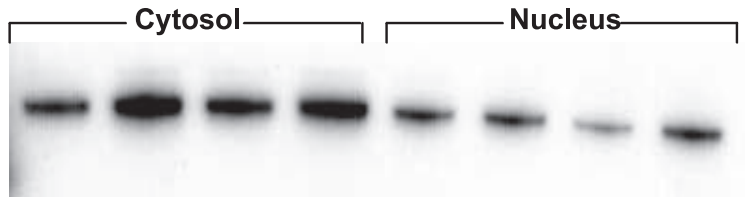

C $\quad 5 \mathrm{~Hz}$
$5 \mathrm{~Hz} \quad 5 \mathrm{~Hz}$ $+\mathrm{APV}+\mathrm{APV}$ + Bic
Figure 3. Bicuculline restores immunostaining for $p$-ERK blocked by NMDAR antagonists. $\boldsymbol{A}, \mathrm{p}$-ERK staining in response to "control" $5 \mathrm{~Hz}$ stimulation is blocked with $50 \mu \mathrm{m} \mathrm{APV} \mathrm{(+APV)} \mathrm{or} 20 \mu \mathrm{m}$ CPP (+ CPP) but is restored when $10 \mu \mathrm{m}$ bicuculline (+Bic.) is used in addition to the NMDAR antagonists. $\boldsymbol{B}$, Experiments similar to those in $\boldsymbol{A}$, except that $100 \mathrm{~Hz}$ synaptic stimulation was used instead of $5 \mathrm{~Hz}$. Stratum pyramidale and stratum radiatum in area CA1 of the hippocampus are shown. The DAB reaction product appears dark, with pyramidal neurons and their apical dendrites showing stain. Note the apical dendrites of pyramidal cells coming off of the cell bodies. Scale bar, $100 \mu \mathrm{m}$. Regions immediately adjacent to the stimulating electrode were chosen for the +APV and + CPP cases to show the few cells that stained positive for $p$-ERK. $p$-ERK staining induced with TBS is not sensitive to APV alone (Dudek and Fields, 2001). C, Left, Immunoblot showing that bicuculline has no effect on basal ERK phosphorylation. Blots were probed with the same antibody as was used in $\boldsymbol{A}$ and $\boldsymbol{B}$. Right, Immunoblot showing an experiment similar to $\boldsymbol{A}$. In both nuclear and cytoplasmic fractions, ERK phosphorylation induced with $5 \mathrm{~Hz}$ stimulation, blocked with $50 \mu \mathrm{M} A \mathrm{PV}$, is restored with $10 \mu \mathrm{m}$ bicuculline. during NMDAR blockade. The difference is likely to be attributable to the fact that synaptic responses typically run down during a $1 \mathrm{~s}$ episode at $100 \mathrm{~Hz}$, perhaps preventing consistent temporal summation beyond the first few pulses. NMDARs thus seem to boost the signal of fatiguing presynaptic terminals to drive action potential firing postsynaptically. Similarly, NMDARs might also 
A

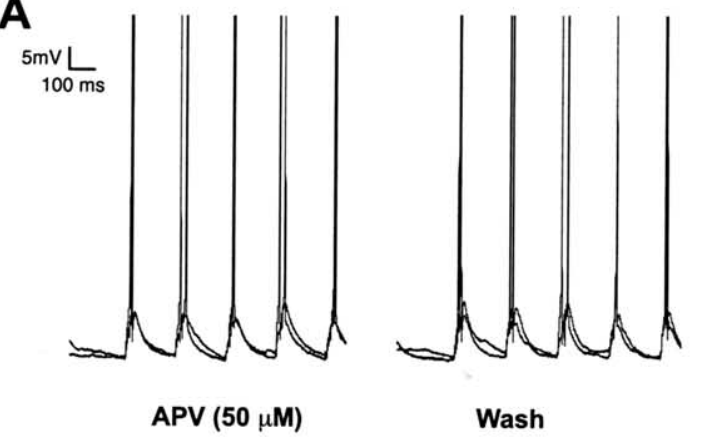

B
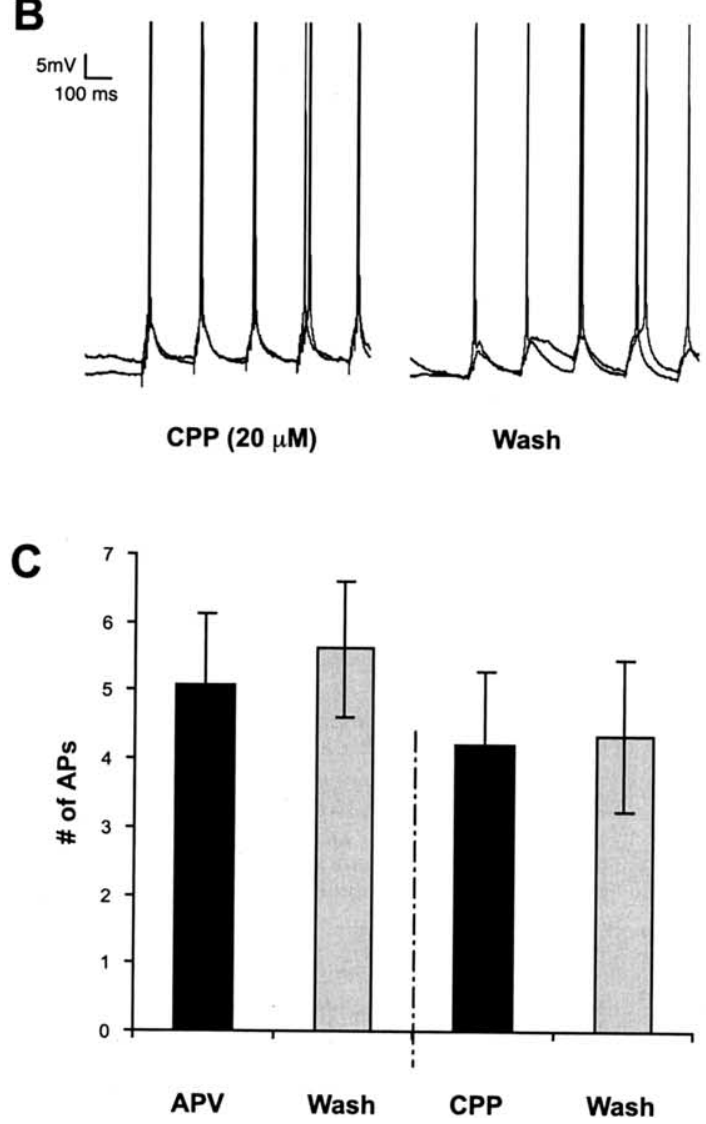

Figure 4. NMDAR antagonists have no effect on action potentials when TBS is used. $\boldsymbol{A}$, Two 1 s recordings during TBS in the presence of APV $(50 \mu \mathrm{M})$ and after washout (Wash) of the drug. $\boldsymbol{B}$, Two superimposed $1 \mathrm{~s}$ recordings during TBS in the presence of CPP $(20 \mu \mathrm{m})$ and after washout (Wash). C, The number of action potentials from experiments like those illustrated in $\boldsymbol{A}$ and $\boldsymbol{B}$ were counted, and the compiled results are shown. Means \pm SEMs from 13 CA1 pyramidal neurons are shown from each frequency. Error bars represent SEMs.

boost the signal of lower frequencies of presynaptic activity, such as $5 \mathrm{~Hz}$, which lack the rapid, repetitive, and summating presynaptic activity of TBS.

Whether the firing patterns of TBS and $100 \mathrm{~Hz}$ differ in the resulting nuclear biochemistry is unknown, although both activate ERK equally (but see Raymond and Redman, 2002; Selcher et al., 2003). However, TBS is very effective at inducing late-LTP with stimulation thresholds very similar to those seen with ERK activation (S. M. Dudek, unpublished observations), between 60 and 80 presynaptic pulses (Dudek and Fields, 2001). Action potentials initiated near the cell body can
A

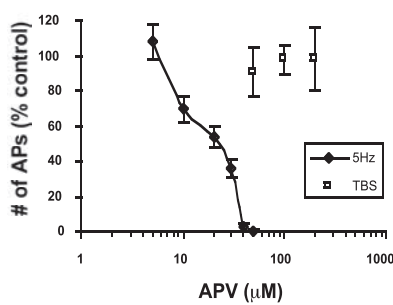

B

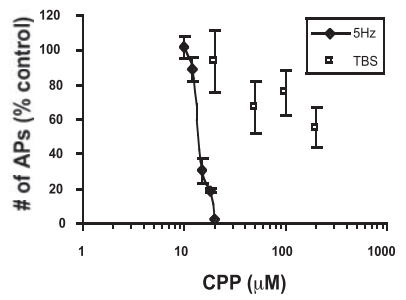

Figure 5. The effects of NMDAR antagonists on action potentials are dose dependent. $\boldsymbol{A}$, Dose-response relationship of APV comparing the effect when $5 \mathrm{~Hz}$ stimulation (closed diamonds) or TBS (open squares) was used ( $n=9,12,10,11,11$, and 10 at $5,10,20,30,40$, and $50 \mu \mathrm{m}$, respectively, for $5 \mathrm{~Hz} ; n=13,10$, and 10 at 50,100 , and $200 \mu \mathrm{m}$, respectively, for TBS). The number of action potentials is presented as a percentage of the predrug control $(5 \mathrm{~Hz})$ or washout control (TBS) conditions. B, Dose-response relationship of CPP ( $n=13,13,13,13$, and 9 at $10,12,15,18$, and $20 \mu \mathrm{m}$, respectively, for $5 \mathrm{~Hz} ; n=9,14,10$, and 9 at $20,50,100$, and $200 \mu \mathrm{m}$, respectively, for TBS) as in $\boldsymbol{A}$.

backpropagate into apical dendrites (Spruston et al., 1995; Johnston et al., 1996), and recent studies on spike-timing dependent plasticity suggest that dendritic action potentials may provide an additional means of achieving the postsynaptic depolarization needed to activate and open NMDAR channels to induce LTP or LTD (Balaban et al., 2004; Dan and Poo, 2004). Accordingly, induction of LTP and/or LTD at the synapse in vivo may have little requirement for postsynaptic firing other than the timing of the presynaptic activity with respect to the postsynaptic firing or bursting. Nuclear events, in contrast, may depend critically on the number and firing rate of postsynaptic action potentials. Synapse specificity would be achieved with the LTP- or LTD-specific tagging of synapses (Sajikumar and Frey, 2004).

One advantage of regulating transcription with action potentials is that large amounts of signal could be generated that are stoichiometrically favorable for transcription. It is unlikely that transcription factors such as nuclear factor $\kappa \mathrm{B}$, for example (Meffert et al., 2003), or a synaptic cargo carried by importin/karyopherin (Thompson et al., 2004) would be produced in quantities sufficient for transcription if produced by only a few synapses, without either the potentiation of many synapses or of an unknown amplifying mechanism. Cell-wide activation of signaling, such as that seen for ERK induced with action potentials, can provide sufficient quantities of active transcription factors on a timescale that supports the rapid induction of some genes [for example, within 5 min for $\operatorname{arc}$ (Guzowski et al., 1999)] and within the time window in which late-phase LTP is sensitive to RNA synthesis inhibitors (Nguyen et al., 1994; Frey et al., 1996). What is the evidence that a synapse-to-nucleus signal is necessary for late-phase LTP? Although some recent data show that importin translocation from synaptic compartments occurs in response to a chemically induced LTP (Thompson et al., 2004), the presence of such a signal in LTP has been mostly inferred based on studies showing that nuclear signaling is sensitive to NMDAR antagonists (Deisseroth et al., 1996; Steward et al., 1998; Matsuo et al., 2000). If the relevant gene expression (transcription and/or translation) relies on action potentials, the effects of NMDAR antagonists could give the mistaken impression of a signal from the synapse if action potentials were inadvertently blocked. Our data support the hypothesis that NMDARs can play a role in nuclear events through their effect on action 
potential generation. Previous evidence has shown that signaling pathways associated with late-LTP can be activated in hippocampal CA1 neurons without synaptic activity; somatic action potentials induced by backfiring the cells are sufficient for phosphorylation of ERK and the cAMP response element-binding protein as well as induction of Zif268 protein (Dudek and Fields, 2002). Furthermore, evidence in support of the idea of action potential-dependent transcriptional events comes from data showing that action potentials alone, in the absence of synaptic activity, are sufficient for the rescue of early-LTP in a synaptic tagging-type experiment (Frey and Morris, 1997; Dudek and Fields, 2002). Although those studies clearly implicated the L-type voltage-sensitive calcium channels in these processes, we find that our stimulation-dependent, NMDARindependent ERK staining is not entirely blocked with nifedipine when bicuculline is used (data not shown). We expect that because bicuculline allows for a greater number of action potentials (or action potentials are better controlled), additional sources of calcium are recruited that are sufficient for ERK activation, such as calcium from internal stores or non-L-type calcium channels. We interpret this to mean that the source of calcium is not critical for ERK activation.

We have now demonstrated that action potentials are not only sufficient but are also necessary for nuclear signaling, in the form of ERK activation, in physiological contexts. Based on these findings, we believe that there are biochemical consequences to blocking NMDARs that are directly related to the role of NMDARs in action potential generation. These findings therefore have important implications for the interpretation of studies showing NMDAR-dependent and -independent forms of learning (Bannerman et al., 1995) and their biochemistries (Cammarota et al., 2000). In the future, the use of theta-burst stimulation or bicuculline could help to distinguish the specific roles that NMDARs have independent from their role in action potential generation.

\section{References}

Abraham WC, Mason SE (1988) Effects of the NMDA receptor/channel antagonists CPP and MK801 on hippocampal field potentials and long-term potentiation in anesthetized rats. Brain Res 462:40-46.

Bading H, Greenberg ME (1991) Stimulation of protein tyrosine phosphorylation by NMDA receptor activation. Science 253:912-914.

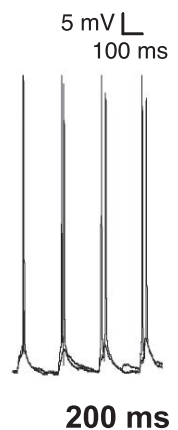

\section{A Within-burst Interval (+50 $\mu \mathrm{M}$ APV)}

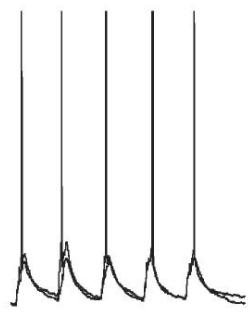

$10 \mathrm{~ms}$

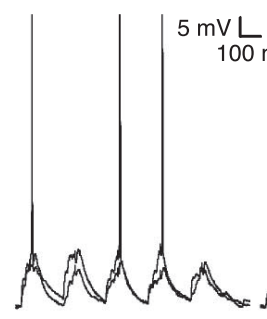

$20 \mathrm{~ms}$

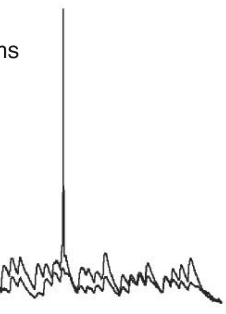

$40 \mathrm{~ms}$

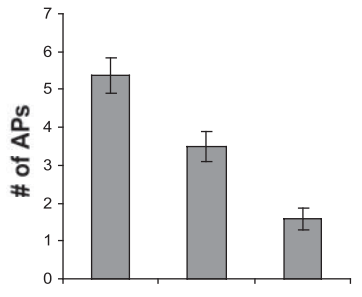

$10 \mathrm{~ms} \quad 20 \mathrm{~ms} \quad 40 \mathrm{~ms}$

\section{B Between-burst Interval (+50 uM APV)}
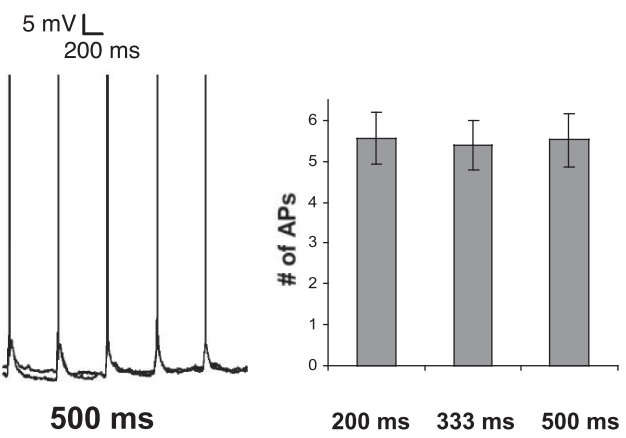

Figure 6. Resistance of TBS-evoked action potentials to APV is caused by temporal summation within bursts. $\boldsymbol{A}$, As intervals between pulses within bursts are increased, the number of action potentials evoked (in $50 \mu \mathrm{M}$ APV) decreases $(n=11)$. Two traces are superimposed showing action potentials induced with TBS consisting of 10,20 , or 40 ms intervals between pulses. $B$, As intervals between bursts are increased, the number of action potentials evoked remains unchanged (in $50 \mu \mathrm{MPV})(n=10)$. Two traces are superimposed showing action potentials induced with TBS consisting of 200, 333, or 500 ms intervals between bursts. Note the different time scales for each set of traces.
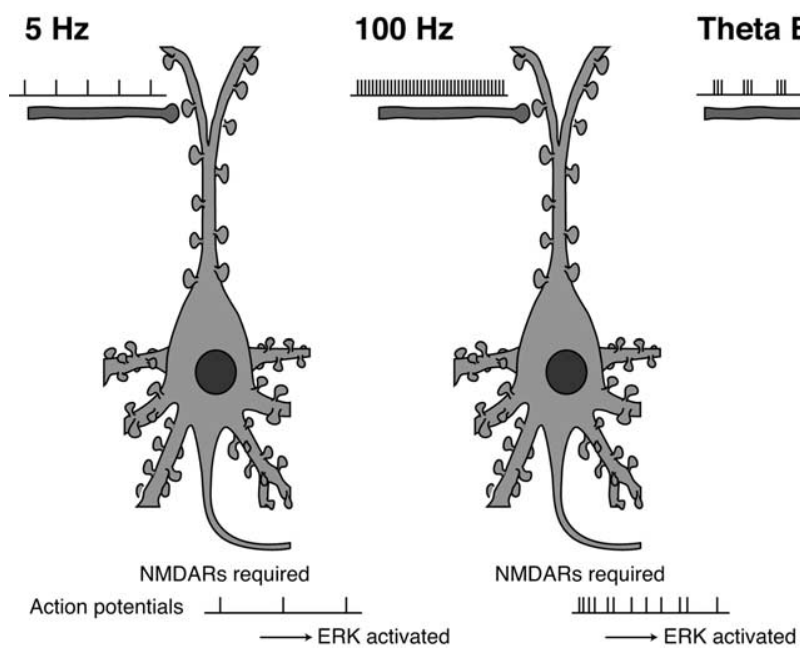

Theta Burst Stim.

Figure 7. Summary of whole-cell ERK activation by synaptic activity. Different presynaptic stimulation ( $5 \mathrm{~Hz}, 100 \mathrm{~Hz}$, or TBS) results in action potentials that are either dependent ( 5 and $100 \mathrm{~Hz}$ ) or independent (TBS) on NMDARs. ERK is activated under either condition, provided action potentials are evoked. Stim., Stimulation.

Balaban P, Chistiakova M, Malyshev A, Volgushev M (2004) Dependence of calcium influx in neocortical cells on temporal structure of depolarization, number of spikes, and blockade of NMDA receptors. J Neurosci Res 76:481-487.

Bannerman DM, Good MA, Butcher SP, Ramsay M, Morris RGM (1995) 
Distinct components of spatial learning revealed by prior training and NMDA receptor blockade. Nature 378:182-186.

Baron C, Benes C, Tan HV, Fagard R, Roisin M-P (1996) Potassium chloride pulse enhances mitogen-activated protein kinase activity in rat hippocampal slices. J Neurochem 66:1005-1010.

Bear M, Kleinschmidt A, Gu Q, Singer W (1990) Disruption of experiencedependent synaptic modifications in striate cortex by infusion of an NMDA receptor antagonist. J Neurosci 10:909-925.

Benveniste M, Meinville J-M, Sernagor E, Mayer ML (1990) ConcentrationJump experiments with NMDA antagonists in mouse cultured hippocampal neurons. J Neurophysiol 63:1373-1384.

Blitz DM, Regehr WG (2003) Retinogeniculate synaptic properties controlling spike number and timing in relay neurons. J Neurophysiol 90:2438-2450.

Blum S, Moore AN, Adams F, Dash PK (1999) A mitogen-activated protein kinase cascade in the CA1/CA2 subfield of the dorsal hippocampus is essential for long-term spatial memory. J Neurosci 19:3535-3544.

Bonansco C, Gonzalez de la Vega A, Gonzalez-Alegre P, Borde M, GarcaSegura LM, Buno W (2002) Tetanic stimulation of Schaffer collaterals induces rhythmic bursts via NMDA receptor activation in rat CA1 pyramidal neurons. Hippocampus 12:434-446.

Burgard EC, Decker G, Sarvey JM (1989) NMDA receptor antagonists block norepinephrine-induced long-lasting potentiation and long-term potentiation in rat dentate gyrus. Brain Res 482:351-355.

Caboche J, Valjent E, Vanhoutte P (2001) Mitogen-activated protein kinase/extracellular signal-regulated kinase induced gene regulation in brain. Mol Neurobiol 23:83-100.

Cammarota M, Bevilaqua LR, Ardenghi P, Paratcha G, Levi de Stein M, Izquierdo I, Medina JH (2000) Learning-associated activation of nuclear MAPK, CREB and Elk-1, along with Fos production, in the rat hippocampus after a one-trial avoidance learning: abolition by NMDA receptor blockade. Brain Res Mol Brain Res 76:36-46.

Dahl D, Burgard EC, Sarvey JM (1990) NMDA receptor antagonists reduce medial, but not lateral, perforant path-evoked EPSPs in dentate gyrus of rat hippocampal slice. Exp Brain Res 83:172-177.

Dan Y, Poo MM (2004) Spike timing-dependent plasticity of neural circuits. Neuron 44:23-30.

Deisseroth K, Bito H, Tsien RW (1996) Signaling from synapse to nucleus: postsynaptic CREB phosphorylation during multiple forms of hippocampal synaptic plasticity. Neuron 16:89-101.

Deisseroth K, Mermelstein PG, Xia H, Tsien RW (2003) Signaling from synapse to nucleus: the logic behind the mechanisms. Curr Opin Neurobiol 13:354-365.

Dudek SM, Fields RD (2001) Mitogen-activated protein kinase/extracellular signal-regulated kinase activation in somatodendritic compartments: roles of action potentials, frequency, and mode of calcium entry. J Neurosci 21:RC122(1-5).

Dudek SM, Fields RD (2002) Somatic action potentials are sufficient for late-phase LTP-related cell signaling. Proc Natl Acad Sci USA 99:3962-3967.

English JD, Sweatt JD (1996) Activation of p42 mitogen-activated protein kinase in hippocampal long term potentiation. J Biol Chem 271:24329-24332.

Frey JU, Morris RG (1997) Synaptic tagging and long-term potentiation. Nature 385:533-536.

Frey U, Frey S, Schollmeier F, Krug M (1996) Influence of actinomycin D, a RNA synthesis inhibitor, on long-term potentiation in rat hippocampal neurons in vivo and in vitro. J Physiol (Lond) 490:703-711.

Guzowski JF, McNaughton BL, Barnes CA, Worley PF (1999) Environment-specific expression of the immediate-early gene Arc in hippocampal neuronal ensembles. Nat Neurosci 2:1120-1124.
Johnston D, Magee JC, Colbert CM, Christie BR (1996) Active properties of neuronal dendrites. Annu Rev Neurosci 19:165-186.

Larson J, Lynch G (1988) Role of $N$-methyl-D-aspartate receptors in the induction of synaptic potentiation by burst stimulation patterned after the hippocampal theta-rhythm. Brain Res 441:111-118.

Larson J, Wong D, Lynch G (1986) Patterned stimulation at the theta frequency is optimal for the induction of hippocampal long-term potentiation. Brain Res 368:347-350.

Martin KC, Casadio A, Zhu H, Yaping E, Rose JC, Chen M, Bailey CH, Kandel ER (1997) Synapse-specific, long-term facilitation of Aplysia sensory to motor synapses: a function for local protein synthesis in memory storage. Cell 91:927-938.

Matsuo R, Murayama A, Saitoh Y, Sakaki Y, Inokuchi K (2000) Identification and cataloging of genes induced by long-lasting long-term potentiation in awake rats. J Neurochem 74:2239.

Meffert M, Chang JM, Wiltgen BJ, Fanselow MS, Baltimore D (2003) NF-KB functions in synaptic signaling and behavior. Nat Neurosci 6:1072-1078.

Miller KD, Chapman B, Stryker MP (1989) Visual responses in adult cat visual cortex depend on $N$-methyl-D-aspartate receptors. Proc Natl Acad Sci USA 86:5183-5187.

Nguyen PV, Abel T, Kandel ER (1994) Requirement of a critical period of transcription for induction of a late phase of LTP. Science 265:1104-1107.

Raymond CR, Redman SJ (2002) Different calcium sources are narrowly tuned to the induction of different forms of LTP. J Neurophysiol 88:249-255.

Richardson GB, Messer C (1995) Effect of composition of experimental solutions on neuronal survival during rat brain slicing. Exp Neurol 131:133-143.

Rosenblum K, Futter M, Voss K, Erent M, Skehel PA, French P, Obosi L, Jones MW, Bliss TV (2002) The role of extracellular regulated kinases I/II in late-phase long-term potentiation. J Neurosci 22:5432-5441.

Sajikumar S, Frey JU (2004) Late-associativity, synaptic tagging, and the role of dopamine during LTP and LTD. Neurobiol Learn Mem 82:12-25.

Selcher JC, Weeber EJ, Nekrasova CJ, Landreth GE, Sweatt JD (2003) A role for ERK MAP kinase in physiologic temporal integration in hippocampal area CA1. Learn Mem 10:26-39.

Sillito AM, Murphy PC, Salt TE, Moody CI (1990) Dependence of retinogeniculate transmission in cat on NMDA receptors. J Neurophysiol 63:347-355.

Spruston N, Schiller Y, Stuart G, Sackmann B (1995) Activity-dependent action potential invasion and calcium influx into hippocampal CA1 dendrites. Science 268:297-300.

Steward O, Wallace CS, Lyford GL, Worley PF (1998) Synaptic activation causes the mRNA for the IEG Arc to localize selectively near activated postsynaptic sites on dendrites. Neuron 21:741-751.

Thiels E, Kanterewicz BI, Norman ED, Trzaskos JM, Klann E (2002) Longterm depression in the adult hippocampus in vivo involves activation of extracellular signal-regulated kinase and phosphorylation of Elk-1. J Neurosci 22:2054-2062.

Thomas MJ, Watabe AM, Moody TD, Makhinson M, O’Dell TJ (1998) Postsynaptic complex spike bursting enables the induction of LTP by theta frequency synaptic stimulation. J Neurosci 18:7118-7126.

Thompson KR, Olofsdotter OK, Chen DY, Zhao Y, O’Dell TJ, Martin KC (2004) Synapse to nucleus signaling during long-term synaptic plasticity: a role for the classical active nuclear import pathway. Neuron 44:997-1009.

Thummler S, Dunwiddie TV (2000) Adenosine receptor antagonists induce persistent bursting in the rat hippocampal CA3 region via an NMDA receptor-dependent mechanism. J Neurophysiol 83:1787-1795. 